\title{
Localised Tuneable Composition Single Crystal Silicon-Germanium-on-Insulator for Low Cost Devices
}

\author{
Callum G. Littlejohns, ${ }^{1,2}$ Thalia Dominguez Bucio, ${ }^{2}$ Milos Nedeljkovic, ${ }^{2}$ Hong Wang, \\ Goran Z. Mashanovich, ${ }^{2}$ Graham T. Reed, ${ }^{2}$ and Frederic Y. Gardes ${ }^{2}$ \\ ${ }^{1}$ Silicon Technologies Centre of Excellence, Nanyang Technological University, 50 Nanyang Avenue, Singapore 639798 \\ ${ }^{2}$ Optoelectronics Research Centre, Building 53, University of Southampton, Southampton SO17 1BJ, UK
}

Correspondence should be addressed to Callum G. Littlejohns; clittlejohns@ntu.edu.sg

Received 11 February 2016; Revised 10 May 2016; Accepted 18 May 2016

Academic Editor: Achim Trampert

Copyright (C) 2016 Callum G. Littlejohns et al. This is an open access article distributed under the Creative Commons Attribution License, which permits unrestricted use, distribution, and reproduction in any medium, provided the original work is properly cited.

\begin{abstract}
The realisation of high quality silicon-germanium-on-insulator (SGOI) is a major goal for the field of silicon photonics because it has the potential to enable extremely low power active devices functioning at the communication wavelengths of $1.3 \mu \mathrm{m}$ and $1.55 \mu \mathrm{m}$. In addition, SGOI has the potential to form faster electronic devices such as BiCMOS transistors and could also form the backbone of a new silicon photonics platform that extends into the mid-IR wavelengths for applications in, amongst others, sensing and telecoms. In this paper, we present a novel method of forming single crystal, defect-free SGOI using a rapid melt growth technique. We use tailored structures to form localised uniform composition SGOI strips, which are suitable for the state-of-the-art device fabrication. This technique could pave the way for the seamless integration of electronic and photonic devices using only a single, low cost Ge deposition step.
\end{abstract}

\section{Introduction}

Silicon-germanium $\left(\mathrm{Si}_{1-x} \mathrm{Ge}_{x}\right)$ is a well-established material in the photonics and electronics industries. It is fully miscible across its entire composition range, therefore enabling the tuning of properties such as the lattice constant [1] and band gap [2], between those of bulk Ge and bulk Si. SiGe alloys can therefore be either optically transparent or optically absorbing at telecoms wavelengths $(1.3 \mu \mathrm{m}$ and $1.55 \mu \mathrm{m})$, depending on its composition. This results in the potential for the fabrication of active optical devices for both modulation $[3,4]$ and detection [5-7]. Additionally, SiGe also has a higher electron mobility and higher hole mobility relative to Si [8], potentially leading to faster electronic devices such as bipolar complementary metal-oxide semiconductor (BiCMOS) transistors $[9,10]$. Tuning of the SiGe lattice constant also enables lattice matching for epitaxial III-V growth [11-13].

Conventional methods of growing SiGe directly on $\mathrm{Si}$ include reduced pressure chemical vapour deposition (RPCVD) [14], low energy plasma enhanced chemical vapour deposition (LEPECVD) [15], and molecular beam epitaxy (MBE) [16]. However, due to the $4.2 \%$ lattice mismatch between $\mathrm{Si}$ and $\mathrm{Ge}$, these methods inevitably introduce high defect densities at the growth interface.

An alternative method of growing SiGe, this time on a localised insulator, is rapid melt growth (RMG). This technique was invented in the 1960s [17] and further developed in the 1970s for the fabrication of LEDs [18], detectors [19], and laser diodes [20]. Originally used for III-V crystal growth, RMG was pioneered by Liu et al. [21] for localised germanium-on-insulator (GOI) growth in 2004. Since then, it has been studied by various groups [22-27] and adapted for silicon-germanium-on-insulator (SGOI) growth [28-37].

A localised SGOI platform paves the way for the monolithic integration of electronic devices with photonic devices on Si substrates, providing a complementary solution to the silicon-on-insulator (SOI) substrates generally used for photonic devices [38]. Process flexibility, CMOS compatibility, and cost are all reasons why the migration of silicon photonic devices to $\mathrm{Si}$ substrates is of major interest. The use of dielectric optical waveguides [39], with localised buried $\mathrm{SiO}_{2}$ 
layers for optical confinement, can enable such a platform. Alternative techniques for fabricating localised SGOI include Ge condensation [40] and layer transfer using a donor wafer [41].

The RMG technique relies on the fact that $\mathrm{Si}$ has a higher melting point than $\mathrm{Ge}\left(1414^{\circ} \mathrm{C}\right.$ and $938^{\circ} \mathrm{C}$, resp. [42]). This means that, at temperatures greater than $938^{\circ} \mathrm{C}$, the $\mathrm{Ge}$ is melted whilst the Si remains solid, maintaining its crystal structure, so that it can act as a template for the recrystallisation of the liquid Ge. Due to the nature of the lateral regrowth upon an insulator, extremely low threading dislocation densities (TDD) can be achieved, potentially leading to improved device characteristics. Additionally, during the melting phase, Si diffuses from the seed into the Ge layer, leading to the formation of SiGe layers from an initial Ge deposition. This deposition is noncritical since it will subsequently be melted to initiate regrowth. Therefore, fabrication complexity is significantly reduced, since the initial Ge deposition does not need to be modified to achieve a different SiGe composition and can be performed using any low cost technique.

However, until now, this RMG technique has resulted in graded SiGe composition profiles or only very high $\mathrm{Ge}$ composition uniform areas. In this paper, we firstly describe the RMG fabrication process and subsequently present a novel method for realising uniform composition, tuneable localised SGOI layers, demonstrating a large range of SiGe compositions.

\section{Materials and Methods}

Fabrication was carried out on 6-inch (100) Si wafers. Firstly, a $50 \mathrm{~nm} \mathrm{SiO}_{2}$ insulating layer is deposited using plasma enhanced chemical vapour deposition (PECVD), upon which the lateral regrowth occurs. Alternatively, a $\mathrm{Si}_{3} \mathrm{~N}_{4}$ layer can be used. The layer thickness can also be varied to satisfy the design requirements. This layer was subsequently densified in a rapid thermal annealer (RTA) to reduce the amount of trapped hydrogen in the $\mathrm{SiO}_{2}$ film. This prevents outgassing during subsequent annealing steps, which could lead to void formation in the SiGe as it recrystallises. Seed areas are then formed into this insulating layer using a dilute $(20: 1) \mathrm{HF}$ wet etch in order to expose the underlying $\mathrm{Si}$, which acts as a template for the SiGe recrystallisation process. Next, nonselective $400 \mathrm{~nm}$ Ge growth was performed by PECVD using $\mathrm{GeH}_{4}(10 \%)$ diluted in $\mathrm{H}_{2}(2.5 \mathrm{sccm})$ and $\mathrm{H}_{2}$ $(100 \mathrm{sccm})$, at a temperature of $250^{\circ} \mathrm{C}$. More details of this Ge growth can be found in [36]. Prior to Ge growth, an HF dip was performed to remove the native $\mathrm{SiO}_{2}$ layer formed on the Si surface and also to terminate the dangling bonds at the $\mathrm{Si}$ surface with $\mathrm{H}$, preventing the formation of a native $\mathrm{SiO}_{2}$ during transfer to the $\mathrm{Ge}$ growth chamber. This HF last approach eliminates the need for a high temperature prebake (to remove the native $\mathrm{SiO}_{2}$ ) prior to epitaxial Ge growth [45]. Figure 1 shows a cross section scanning electron microscope (SEM) image at the seed edge after the Ge growth, confirming nonselective growth.

The Ge was then inductively coupled plasma (ICP) etched into the desired structures, ensuring that one end of the Ge pattern overlapped with the $\mathrm{Si}$ seed area. A $1 \mu \mathrm{m} \mathrm{SiO}{ }_{2}$

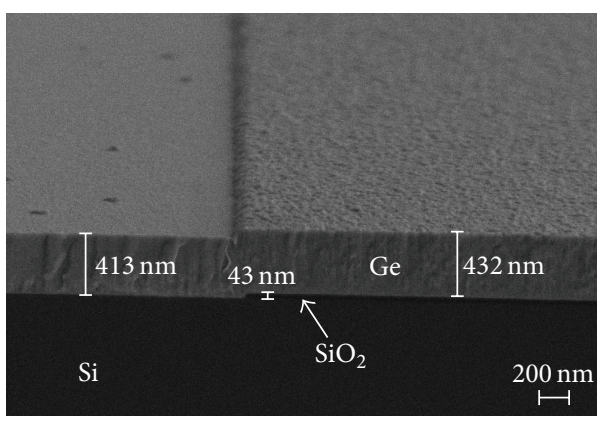

FIGURE 1: Cross section SEM image of Ge growth at the seed edge.

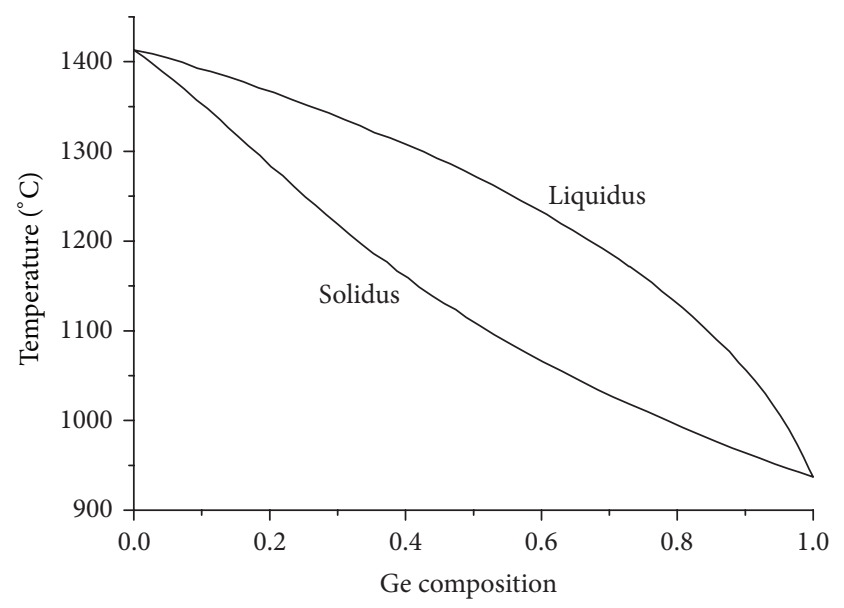

FIgURE 2: SiGe phase diagram, adapted from [43].

layer was then deposited by PECVD to encapsulate the Ge structures to contain the liquid Ge during subsequent melting in an RTA. In this melting step, the temperature of the RTA was stabilised at approximately $500^{\circ} \mathrm{C}$, before ramping up to the peak temperature at a rate of approximately $100^{\circ} \mathrm{C} / \mathrm{s}$. The temperature was held at its peak for 1 second, before cooling naturally (i.e., switching off the RTA lamps).

2.1. Regrowth Mechanism. When the temperature is raised above the melting point of $\mathrm{Ge}, \mathrm{Si}$ diffusion from the seed is greatly enhanced [46], leading to the formation of a SiGe liquid. Upon cooling, regrowth is then initiated at the Si seed, firstly because the substrate acts as a heat sink and secondly because the slightly higher Si composition in the seed area results in solidification at a slightly higher temperature, as defined by the SiGe phase diagram shown in Figure 2 . Epitaxial regrowth then propagates laterally along the SiGe structure. It has been shown that all threading dislocations are confined to the seed area by a defect necking mechanism, as shown in [36].

2.2. Material Characterisation. The SiGe material has been characterised using $532 \mathrm{~nm}$ Raman spectroscopy to determine its composition. Raman spectroscopy is a nondestructive, microscale technique, which does not require any sample preparation, and is commonly used for this type of analysis $[23,47-49]$. A 50x objective lens was used, resulting in a 
laser spot size of approximately $0.5 \mu \mathrm{m}$. The data gathered by this technique relates to the near-surface information only because a laser of this wavelength has a penetration depth of several tens of nanometers in high Ge percentage $\mathrm{SiGe}$ (depending on the composition [50]). It is therefore possible that there is a nonuniform vertical distribution of $\mathrm{Ge}$. However, this is predicted to be negligible [33], especially in areas away from the seed, since the epitaxial regrowth front propagates laterally.

Lateral scans were performed along each structure, resulting in a series of Raman spectra, each of which can be analysed to determine the SiGe composition at that point. Two characteristic peaks can be seen in each spectrum: the first at a wavenumber between $280 \mathrm{~cm}^{-1}$ and $300 \mathrm{~cm}^{-1}$, corresponding to the $\mathrm{GeGe}$ mode vibrations, and the second at a wavenumber between $390 \mathrm{~cm}^{-1}$ and $410 \mathrm{~cm}^{-1}$, corresponding to the SiGe mode vibrations. The wavenumber of each mode depends on the composition of the measured material, which therefore enables the composition to be determined using the peak location [48, 51]. Shin et al. [51] showed that the unstrained GeGe Raman peak location, $\omega_{\mathrm{GeGe}}$, can be calculated by

$$
\omega_{\mathrm{GeGe}}=280.8+19.37 x \text {, }
$$

where $x$ is the Ge concentration. However, this type of measurement is complicated by the fact that the peak location also depends on strain [52-54]. In fact, an in-plane strain of $1 \%$ will induce a peak shift of approximately $3.85 \mathrm{~cm}^{-1}$ [52]. Nevertheless, as proposed by Mooney et al. [47] (2), the composition can be determined using the relative intensities of the SiGe and GeGe peaks, independently of the strain:

$$
\frac{I(\mathrm{SiGe})}{I(\mathrm{GeGe})}=\frac{2(1-x)}{k x},
$$

where $I(\mathrm{SiGe})$ and $I(\mathrm{GeGe})$ are the integrated intensities of the $\mathrm{SiGe}$ and $\mathrm{GeGe}$ modes, respectively, $x$ is the $\mathrm{Ge}$ concentration, and $k$, which is dependent on the excitation wavelength, is an experimental setup specific constant that can be determined from samples of known composition. Using this particular setup, $k$ was determined to be 1.2.

\section{Results and Discussion}

3.1. Composition Profiles of Straight Strips. After RMG was performed, the encapsulating $\mathrm{SiO}_{2}$ was removed in $20: 1 \mathrm{HF}$ acid to enable SEM viewing of the SiGe structures. Figure 3 shows an array of strip widths, each with a length of $200 \mu \mathrm{m}$.

From this image, it is evident that there is a maximum strip width, above which RMG becomes unstable. Closer inspection reveals the maximum width to be approximately $5 \mu \mathrm{m}$, using the fabrication process described above. The liquid $\mathrm{SiGe}$ agglomerates at widths greater than $5 \mu \mathrm{m}$, possibly due to the high interface energy between $\mathrm{SiO}_{2}$ and $\mathrm{SiGe}$ [25], resulting in dewetting of the $\mathrm{SiO}_{2}$ surface when the $\mathrm{SiGe}$ is in the liquid phase. Agglomeration is suppressed at strip widths less than $5 \mu \mathrm{m}$, so only strips with a width of $5 \mu \mathrm{m}$ or less are investigated in detail.

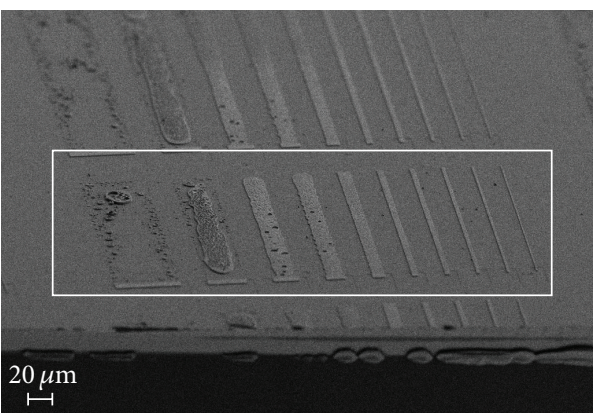

FIGURE 3: SEM image of RMG straight strips showing agglomeration at large strip widths.

Figure 4(a) shows the composition profile as a function of distance from the Si seed for a variety of strip lengths. The annealing temperature of the $\mathrm{SiGe}$ has been estimated from the $\mathrm{SiGe}$ phase diagram to be $1008^{\circ} \mathrm{C}$, based on the lowest observed Ge composition.

From Figure 4(a), it can be observed that the composition varies in the straight strips, from lower Ge composition at the seed to $100 \% \mathrm{Ge}$ at the distal end of the strip. The composition profile is independent of length (for this range of lengths), which can be seen in Figure 4(b), where the strip length has been normalised by dividing each point by the total length of each respective strip. This graded composition profile is a result of the large separation between the solidus and liquidus curves of the SiGe phase diagram (Figure 2), which therefore results in preferential Si rich solid formation at the regrowth front, with rejection of $\mathrm{Ge}$ into the liquid. The complete mixing model [33], based on analysis originally carried out by Scheil [55], has been added to each curve and is described in the following.

3.2. Segregation Coefficient. The segregation coefficient, $k$, of a SiGe alloy defines the degree of segregation between $\mathrm{Ge}$ and $\mathrm{Si}$. It is defined as the ratio of the Ge solid composition, $C_{S}$, to the Ge liquid composition, $C_{L}$, at any particular temperature:

$$
k=\frac{C_{S}}{C_{L}} .
$$

$k$ can be calculated from the SiGe phase diagram, as shown in Figure 5(a). This is then plotted as a function of the solid Ge composition in Figure 5(b).

As the Ge composition approaches $100 \% \mathrm{Ge}$, the segregation coefficient approaches unity. On the contrary, as the Ge composition decreases, there is a higher degree of segregation. A simple linear fit has been applied between Ge compositions of 0.4 and 1.0, leading to (4) for the segregation coefficient as a function of the Ge solid composition, $C_{S}$ :

$$
k=\frac{C_{S}}{C_{L}}=0.78 C_{S}+0.21
$$

In this paper, no Ge compositions lower than 0.4 have been measured, therefore deeming this approximation valid. 


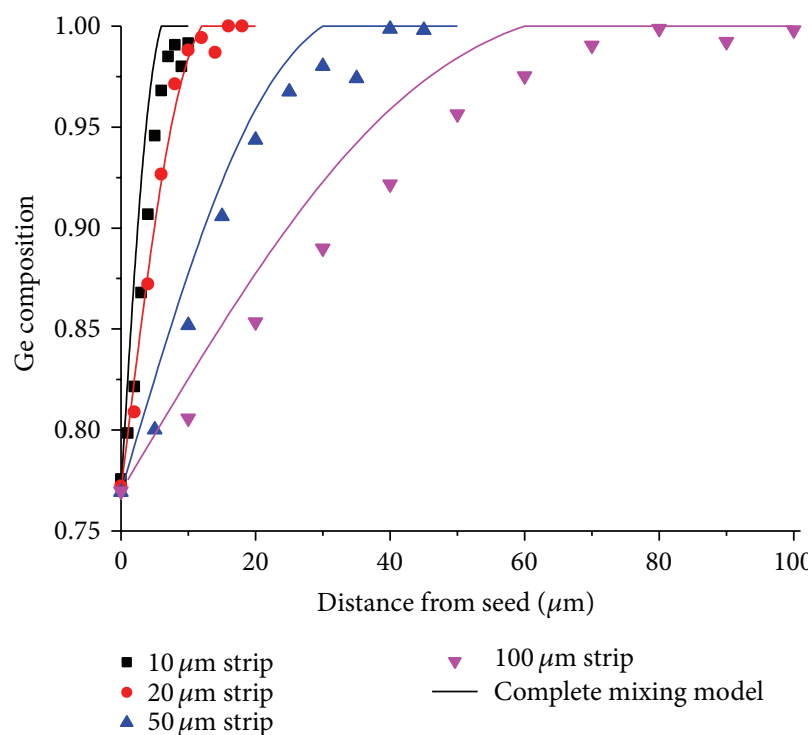

(a)

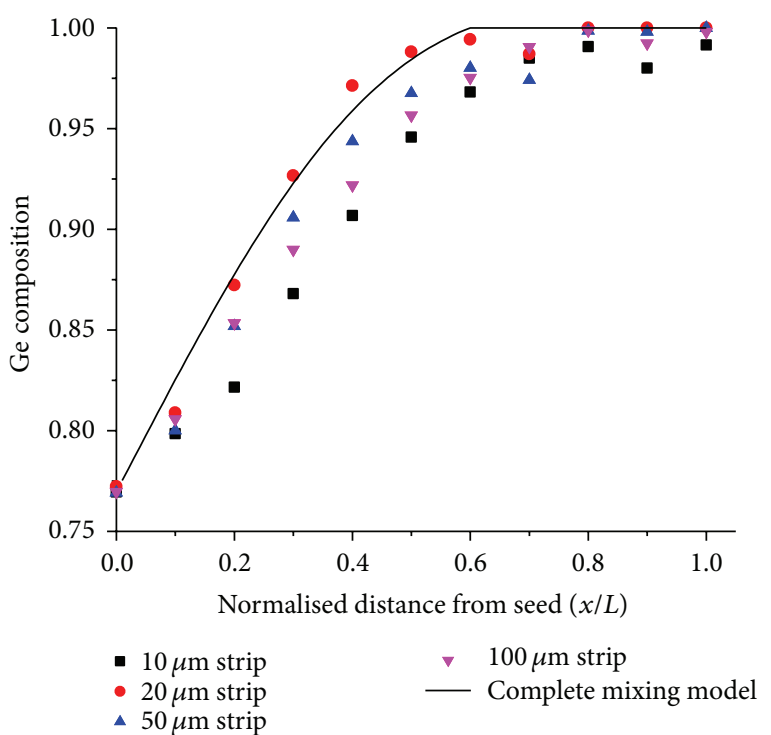

(b)

FIGURE 4: SiGe composition profiles for a range of straight strip lengths: (a) as a function of distance from the seed and (b) normalised by dividing the distance from the seed by the total strip length. Strip width $=5 \mu \mathrm{m}$. Estimated maximum SiGe temperature $=1008^{\circ} \mathrm{C}$. The strips have been fitted with the complete mixing model [33].

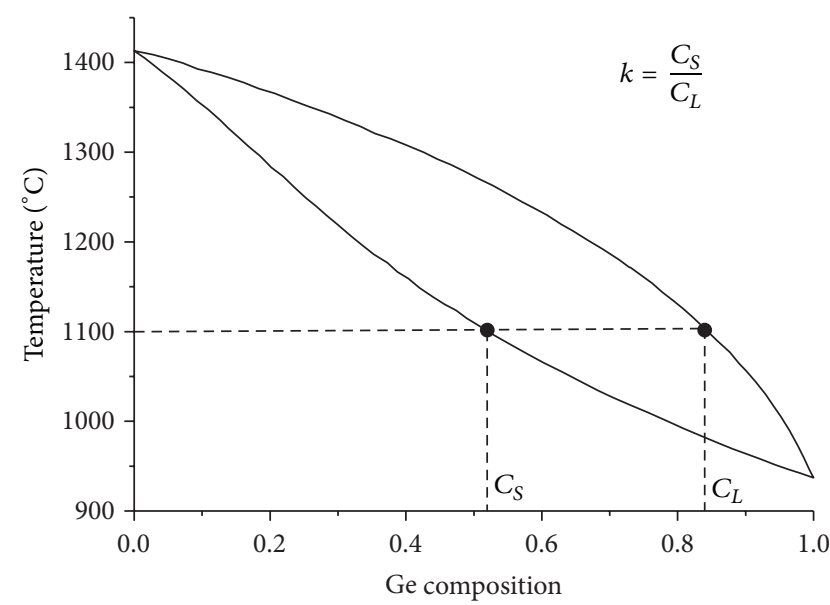

(a)

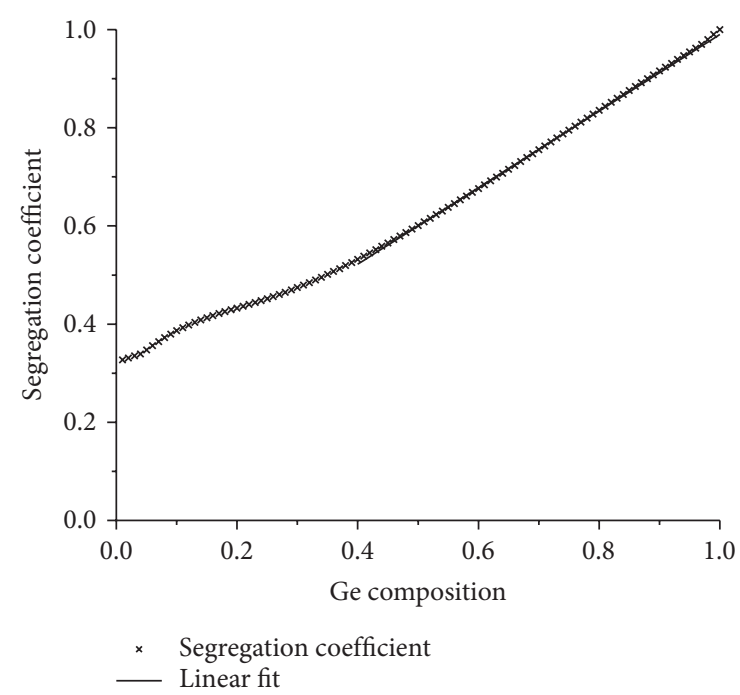

(b)

FIGURE 5: SiGe segregation coefficient. (a) Calculating the segregation coefficient from the phase diagram and (b) SiGe segregation coefficient as a function of $\mathrm{Ge}$ composition. A linear fit has been applied between $\mathrm{Ge}$ compositions of 0.4 and 1.0.

3.3. Scheil Equation. The simplest model of binary alloy solidification is possibly the Scheil equation [55]:

$$
C_{S}=k C_{0}\left(1-f_{S}\right)^{k-1},
$$

where $f_{S}$ is the fraction of the strip that has solidified and $C_{0}$ is the average Ge composition in the liquid at the point when solidification commences. The Scheil equation makes 3 assumptions:

(1) There is complete mixing of $\mathrm{Ge}$ and $\mathrm{Si}$ in the melt, resulting in a uniform composition.
(2) There is no diffusion in the solid.

(3) $k$ is constant. This is only valid if the phase diagram is simplified to become straight lines, which is approximately true for small composition ranges.

The segregation coefficient (3) determines the solid composition to commence at $k C_{0}$, since the liquid is at a uniform composition of $C_{0}$. The fact that the Scheil equation assumes complete mixing in the melt means that it is only accurate when the regrowth front propagation speed is slow, therefore 


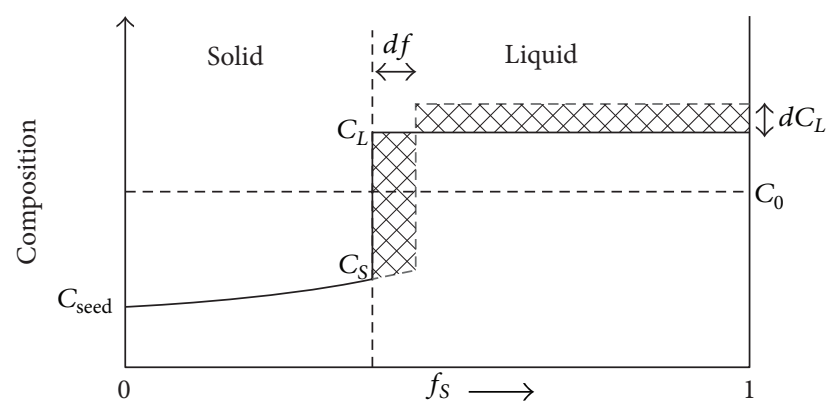

FIGURE 6: Diagram showing the derivation of the complete mixing model.

providing enough time for the rejected $\mathrm{Ge}$ at the regrowth front to fully diffuse into the melt.

3.4. Complete Mixing Model. A more complete version of the Scheil equation is the complete mixing model [33]. This model does not assume a constant value of $k$. Assumptions (1) and (2) (listed above) still remain valid. In this paper, the simplified linear equation for the segregation coefficient (4) has been used.

To derive the complete mixing model, we must consider a small progression of the regrowth front, as shown in Figure 6.

Here, the solid line shows the composition profile at any given point in time, with the solid composition on the lefthand side and the liquid composition on the right-hand side. The dashed line shows the composition profile after a small progression of the regrowth front, $d f$. Ge is rejected from the solid into the liquid at the regrowth front. Therefore, conservation of mass enables equating the amount of $\mathrm{Ge}$ in the two hashed areas:

$$
\left(C_{L}-C_{S}\right) d f=\left(1-f_{S}\right) d C_{L}
$$

The segregation coefficient, $k$, can then be used to replace $C_{S}$. Rearranging (4) gives

$$
C_{S}=\frac{0.21 C_{L}}{1-0.78 C_{L}}
$$

Substituting (7) into (6) and integrating both sides result in

$$
\begin{aligned}
1-f_{S}= & \left(\frac{C_{\text {seed }}}{C_{S}}\right)^{1 / 0.79}\left(\frac{0.79-0.78 C_{S}}{0.79-0.78 C_{\text {seed }}}\right)^{0.21 / 0.79} \\
& \cdot\left(\frac{0.78 C_{S}+0.21}{0.78 C_{\text {seed }}+0.21}\right)
\end{aligned}
$$

where $C_{\text {seed }}$ is the solid composition at the edge of the seed. This is the complete mixing model, valid for $\mathrm{Ge}$ compositions between 0.4 and 1.0. The full derivation of the complete mixing model can be found in Appendix D of [56]. This equation is also only valid when the regrowth front propagation speed is low because of the assumption that the melt has a uniform composition (i.e., complete mixing of the rejected $\mathrm{Ge}$ into the melt). In addition, this equation is also valid when the distance left to solidify is low (i.e., a few microns), since complete mixing can still occur.
Figure 4 shows that the complete mixing model is a close match to the measured data, suggesting that, for straight strips of these lengths $(<100 \mu \mathrm{m})$, the regrowth front propagation speed is slow enough to enable complete mixing of the rejected Ge into the melt.

However, for the fabrication of the state-of-the-art devices using this material, it is essential to form uniform composition areas of SGOI. This can be achieved by steadystate solidification, whereby Ge diffusion into the melt is dramatically reduced by increasing the regrowth front propagation speed.

3.5. Steady-State Solidification. In steady-state solidification, there is limited Ge diffusion into the melt. When the regrowth front propagation speed is nonnegligible, this is a more accurate model than both the Scheil equation and the complete mixing model. Here, diffusion of the rejected Ge at the regrowth front is balanced by the propagation of the regrowth front, therefore resulting in a uniform composition profile. Diffusion of Ge is given by Fick's second law:

$$
\frac{d C_{S}}{d t}=D_{L} \frac{d^{2} C_{S}}{d x^{2}}
$$

Also,

$$
\frac{d C_{S}}{d t}=\frac{d C_{S}}{d x} \frac{d x}{d t}=v \frac{d C_{S}}{d x}
$$

where $v$ is the regrowth front propagation speed, $D_{L}$ is the diffusivity of $\mathrm{Ge}$ in liquid $\mathrm{SiGe}$, and $x$ is the length in the liquid with a nonuniform composition. This equation assumes a constant regrowth propagation speed. These equations combine to give

$$
D_{L}\left(\frac{d^{2} C_{S}}{d x^{2}}\right)-v\left(\frac{d C_{S}}{d x}\right)=0 .
$$

This is a second-order differential equation. Therefore, the solution is of the following form:

$$
C_{S}=\alpha e^{\beta x}+\gamma \text {. }
$$

The first- and second-order differentials of this equation are

$$
\begin{aligned}
\frac{d C_{S}}{d x} & =\beta \alpha e^{\beta x}, \\
\frac{d^{2} C_{S}}{d x^{2}} & =\beta^{2} \alpha e^{\beta x} .
\end{aligned}
$$

Substituting these two equations into (11) gives (note the change in sign since Ge diffusion and regrowth are in the same direction)

$$
D_{L} \beta^{2} \alpha e^{\beta x}+v \beta \alpha e^{\beta x}=0,
$$

which simplifies to

$$
\beta=\frac{-v}{D_{L}} .
$$




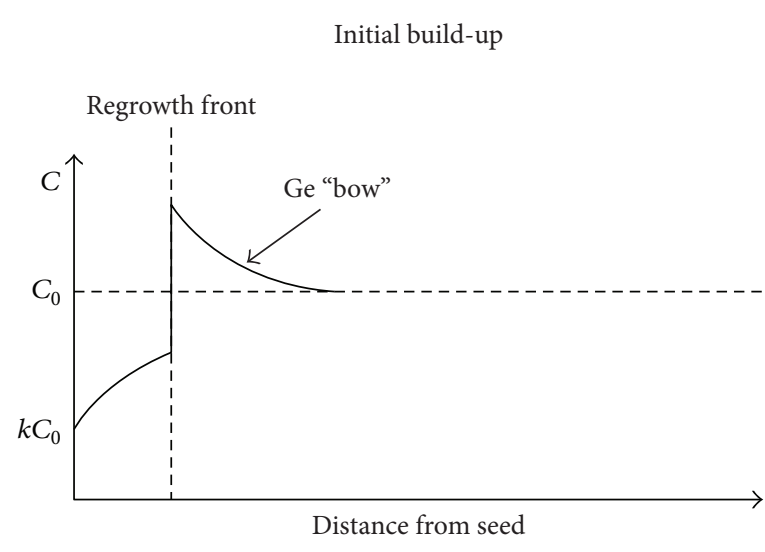

(a)

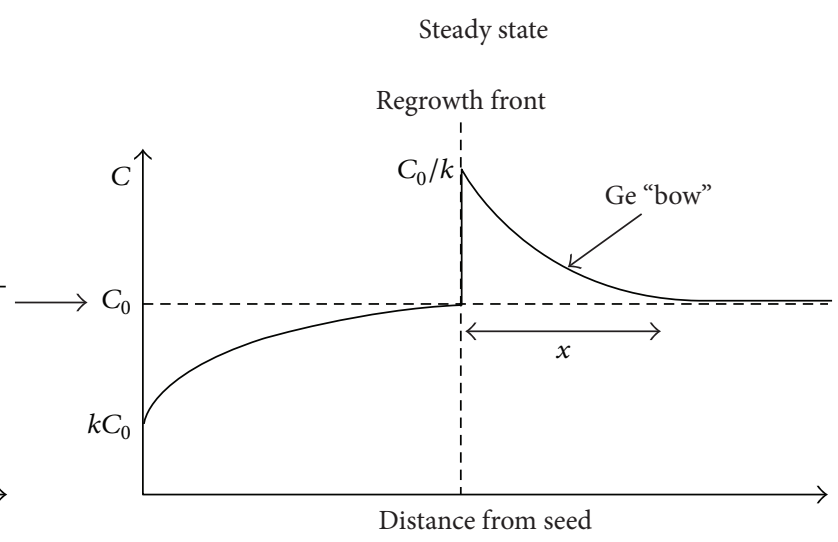

(b)

FIGURE 7: Diagram showing steady-state solidification. (a) Initial build-up and (b) steady-state solidification.

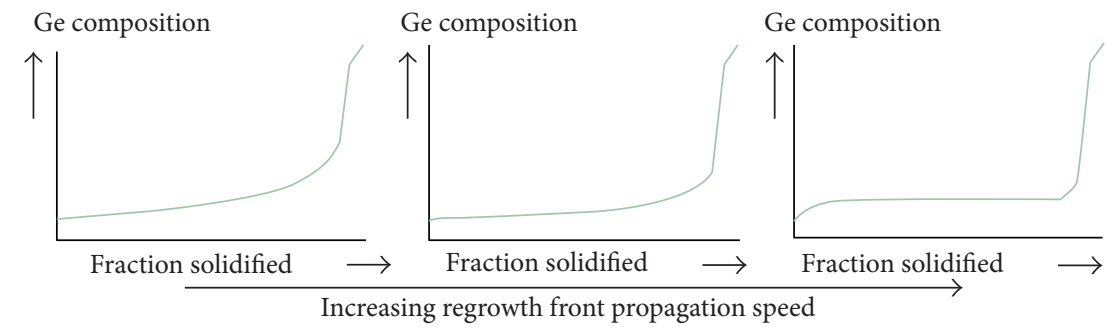

(a)

(b)

(c)

FIGURE 8: Simulations of Ge composition profiles formed by steady-state solidification using the University of Cambridge software [44] showing increasing regrowth front propagation speed from (a) to (c).

Substituting this into the general solution of a second-order differential equation results in

$$
C_{S}=\alpha e^{\left(-x /\left(D_{L} / v\right)\right)}+\gamma .
$$

The boundary conditions for this equation are $C_{S}=C_{0}$ when $x=\infty$ and $C_{S}=C_{L}$ when $x=0$. Substituting the boundary conditions into (16) results in

$$
\gamma=C_{0}
$$

And, therefore,

$$
\alpha=C_{L}-C_{0} .
$$

Substituting these back into (16),

$$
C_{S}=C_{0}+\left(C_{L}-C_{0}\right) e^{\left(-x /\left(D_{L} / v\right)\right)},
$$

which becomes

$$
C_{S}=C_{0}+\frac{C_{0}(1-k)}{k} e^{\left(-x /\left(D_{L} / v\right)\right)} .
$$

This is the steady-state equation for alloy solidification, which is detailed in Figure 7.

In steady-state solidification, the regrowth front propagation speed is faster than that in the complete mixing model. Therefore, as the Ge is rejected from the solid at the regrowth front, there is insufficient time for it to fully diffuse into the bulk melt. This results in an initial build-up of a Ge "bow" (Figure 7(a)) at the regrowth front as solidification commences, followed by a steady-state period once the solid Ge composition reaches $C_{0}$ (Figure 7(b)). At this point, the propagating regrowth front effectively sees the same Ge composition in the melt directly ahead of it, independent of the distance from the seed, and therefore a uniform composition is achieved. However, at the distal end of the structure, when $x$ becomes small, completed diffusion is realised and the composition profile follows the complete mixing model.

Steady-state solidification composition profiles can be predicted using the University of Cambridge website "Dissemination of IT for the Promotion of Materials Science" [44] simulation software. Figure 8 shows the predicted composition profile as the regrowth front propagation speed is increased from Figures 8(a)-8(c).

These simulations demonstrate that increasing the regrowth front propagation speed results in a flatter composition profile because the diffusion of the rejected Ge into the melt at the regrowth front is balanced by the propagation of the regrowth front itself.

It is therefore necessary to maximise the regrowth front propagation speed to achieve a uniform composition profile. This can be realised by maximising the cooling rate of the SiGe structures after melting [57]. This cannot be accomplished by altering the RTA cooling process since the lamps 


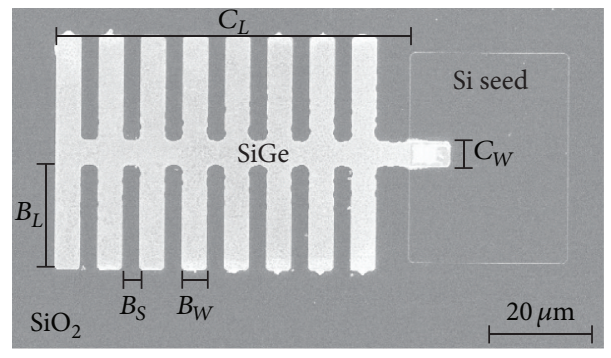

FIGURE 9: SEM image of a tailored tree-like structure. Dimensions are defined as follows: centre strip length $C_{L}$, centre strip width $C_{W}$, branch length $B_{L}$, branch width $B_{W}$, branch separation $B_{S}$, and number of branches $n$.

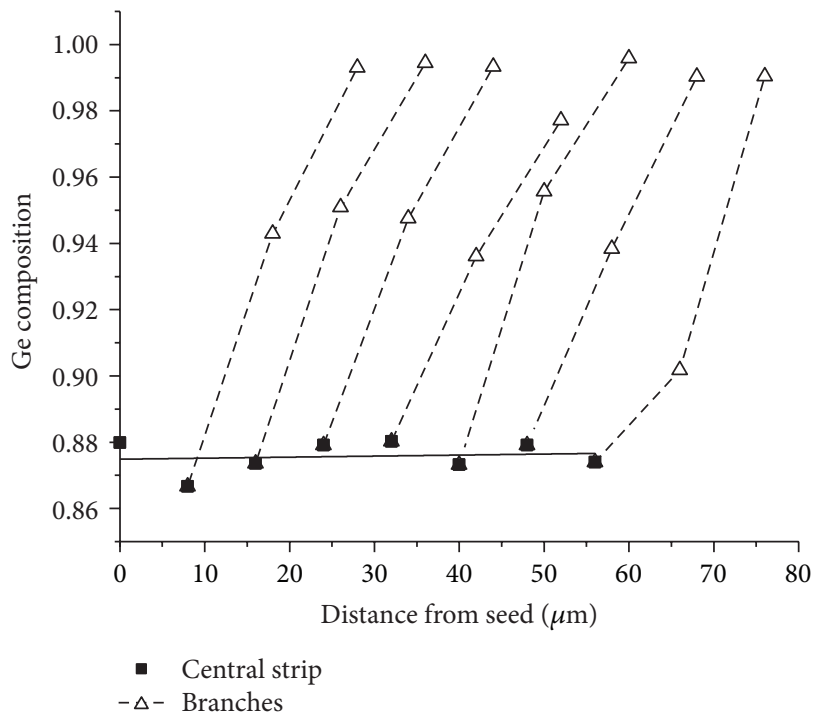

(a)

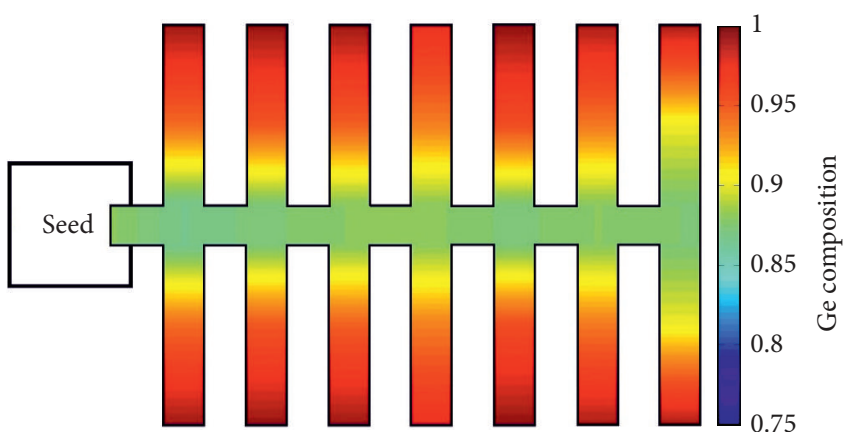

(b)

Figure 10: SiGe composition profile of a tailored tree-like structure. (a) Graph and (b) schematic. $C_{W}=3 \mu \mathrm{m}, C_{L}=65 \mu \mathrm{m}, B_{W}=5 \mu \mathrm{m}, B_{L}$ $=20 \mu \mathrm{m}$, and $B_{S}=3 \mu \mathrm{m}$. The final set of branches have been omitted to display only the uniform composition region. Estimated maximum $\mathrm{SiGe}$ temperature $=975^{\circ} \mathrm{C}$. A linear fit has been added to the central strip.

are already switched off immediately after heating. Therefore, it is necessary to increase the cooling rate by other means.

3.6. SiGe Composition Profiles of Tailored Structures. In order to realise a uniform SiGe composition, a tailored tree-like structure was designed, as shown in Figure 9. Branches were added to the main central strip, which act as radiating elements with the purpose of increasing the cooling rate and therefore the regrowth front propagation speed of the structure.

The composition profile of the complete tree-like structure is shown in Figure 10.

Figure 10 shows that a uniform composition has been achieved in the central strip of a tree-like structure, when the central strip width is $3 \mu \mathrm{m}$. Only the uniform composition area (first $56 \mu \mathrm{m}$, corresponding to 7 out of 8 sets of branches) has been shown. After the first $56 \mu \mathrm{m}$ (penultimate set of branches), the cooling effects of the branches are reduced, and the length of strip left to solidify becomes small, resulting in a nonuniform SiGe composition profile. Each branch exhibits a graded composition profile, suggesting that the central strip solidifies first and then subsequently acts as a seed for the regrowth of the branches.

Figure 11 compares the composition profile of the central strip of a tree-like structure with a straight strip with the same dimensions.

3.7. SiGe Composition Tuning by Annealing Temperature. It is desirable to be able to tune the SiGe composition in the central strip, without the need to alter the initial Ge growth conditions, so that this RMG technique can be used for a whole range of device designs. This can be achieved simply by modifying the annealing temperature, according to the phase diagram, as shown in Figure 12.

Figure 12(a) shows that a uniform composition is realised for all the demonstrated annealing temperatures. A higher annealing temperature results in greater $\mathrm{Si}$ diffusion into the structure before solidification commences and therefore leads to a lower Ge composition upon solidification. This is demonstrated in Figure 12(b), where the average Ge composition in the central strip of the tree-like structures has been plotted as a function of annealing temperature. 

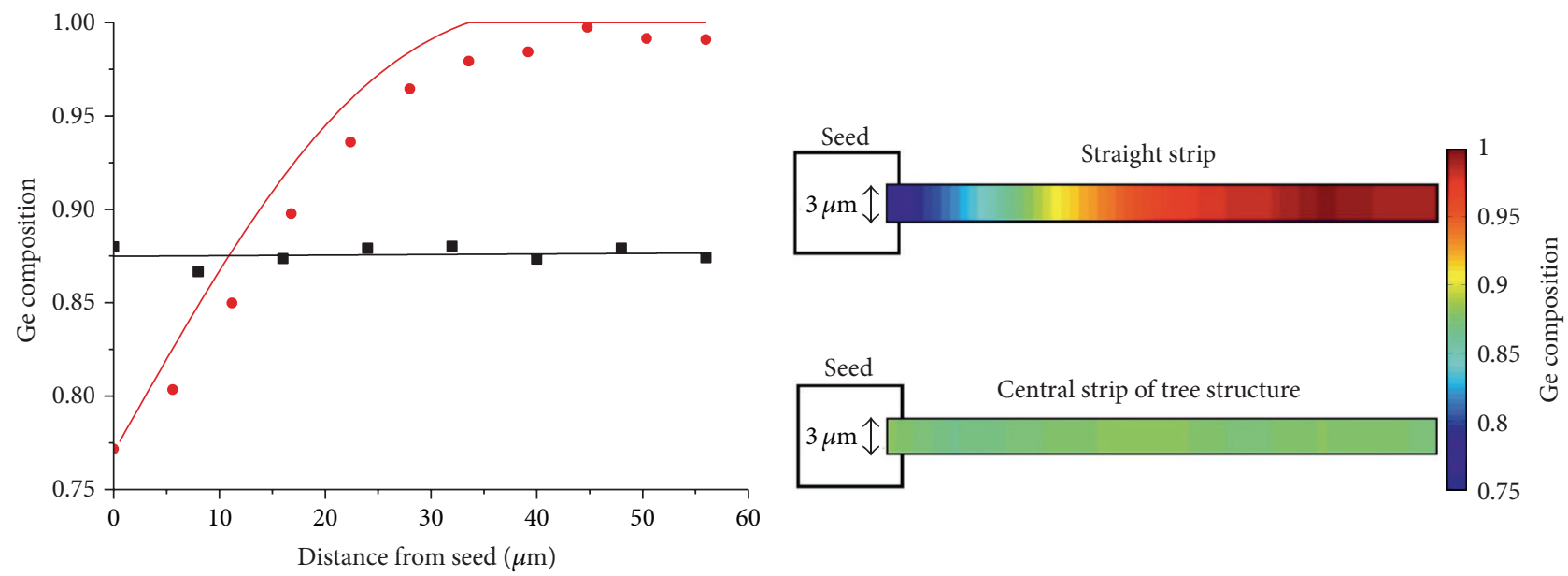

- Central strip of tree structure

- Straight strip

- Complete mixing model

(a)

(b)

FIGURE 11: Comparison of SiGe composition profiles of the central strip of a tailored tree-like structure and a straight strip. (a) Graph and (b) schematic. The final set of branches have been omitted from the central strip of the tailored tree-like structure to display only the uniform composition region. The straight strip data has been normalised from Figure 4 to match the length of the tailored tree-like structure. The complete mixing model has been fitted to the straight strip data [33]. A linear fit has been added to the tailored tree-like structure data.
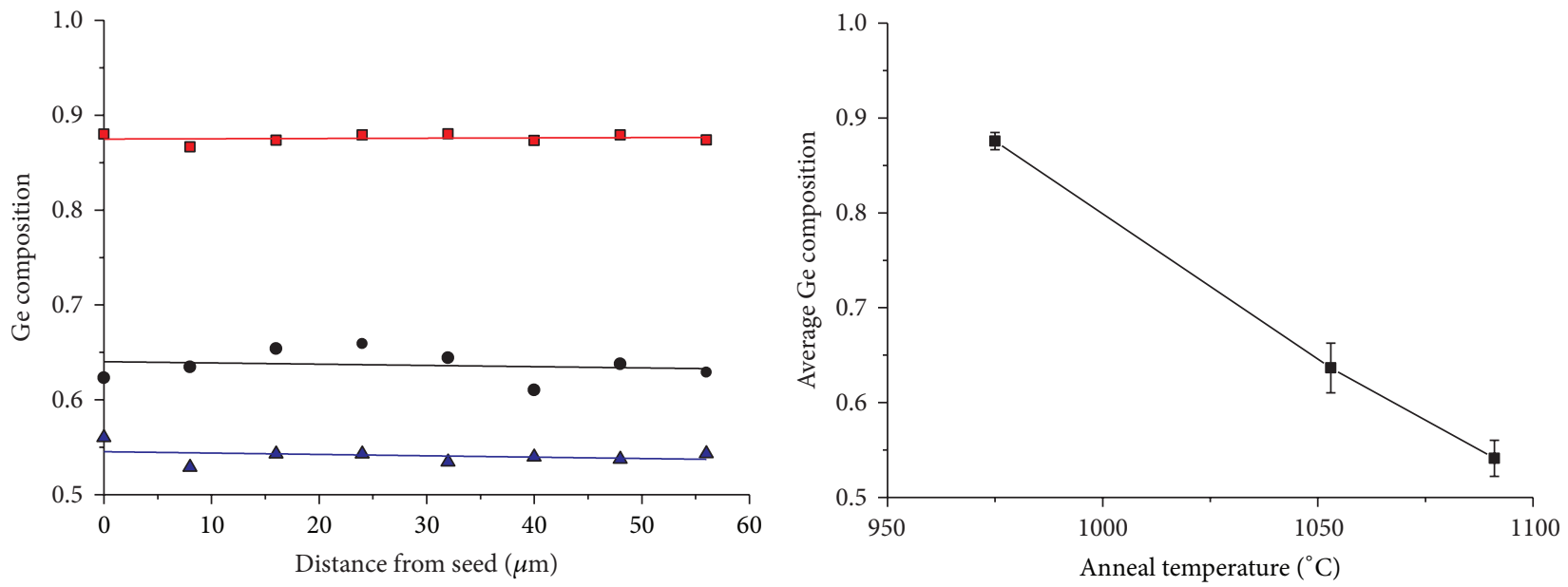

Anneal temperature:

- $975^{\circ} \mathrm{C}$

- $1053^{\circ} \mathrm{C}$

- $1091^{\circ} \mathrm{C}$

(a)

(b)

FIGURE 12: SiGe composition tuning by varying the RMG annealing temperature. (a) Composition profiles in the central strips of tailored tree-like structures annealed at a range of temperatures. A linear fit has been added to each data set and (b) average Ge composition along the central strip of a tailored tree-like structure as a function of annealing temperature. Only the uniform region up to $56 \mu \mathrm{m}$ has been considered. The error bars show the maximum and minimum values observed along each strip. $C_{W}=3 \mu \mathrm{m}, C_{L}=65 \mu \mathrm{m}, B_{L}=20 \mu \mathrm{m}, B_{W}=5 \mu \mathrm{m}$, and $B_{S}$ $=3 \mu \mathrm{m}$. 


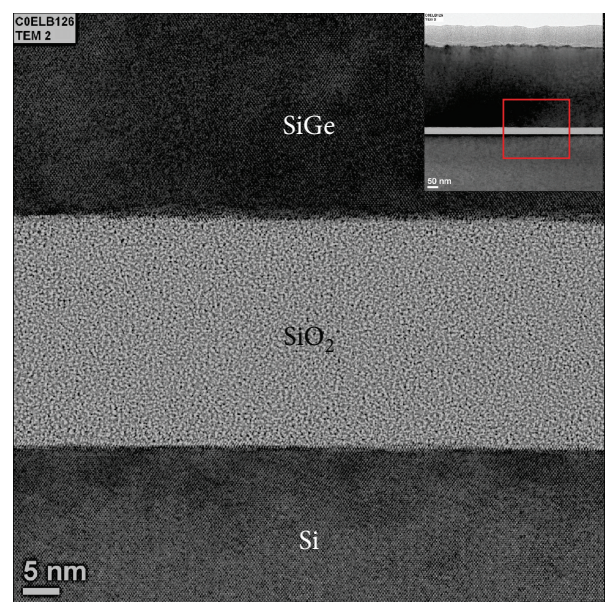

FIGURE 13: High resolution cross section TEM image of RMG SiGe in the central strip of a tree-like structure.

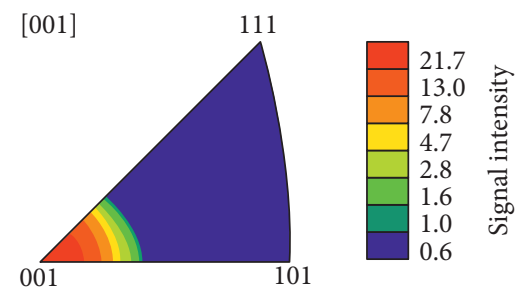

Figure 14: Pole figure of RMG SiGe measured by EBSD. The measured area was approximately $3 \mu \mathrm{m} \times 3 \mu \mathrm{m}$.

3.8. Material Quality Analysis. Transmission electron microscopy (TEM) has been performed to image a cross section of the central strip of a SiGe tree-like structure. Figure 13 confirms single crystal, defect-free SGOI fabrication using this RMG method.

Electron backscatter diffraction (EBSD) measurements were also performed to measure the crystal orientation of the RMG SiGe. Figure 14 shows a pole figure of an area approximately $3 \mu \mathrm{m} \times 3 \mu \mathrm{m}$. The observed data confirms single crystal (001) orientated $\mathrm{SiGe}$, matching the crystal orientation of the Si substrate, as expected. [36].

A more detailed material quality analysis can be found in

\section{Conclusion}

In conclusion, the rapid melt growth technique described here presents a fabrication process for producing localised single crystal SiGe layers of uniform composition, suitable for the state-of-the-art device fabrication. This method enables the simple tuning of the SiGe composition, without the need to alter the initial growth conditions, and uses low cost, readily available CMOS fabrication tools. It offers the potential for the migration of silicon photonics to bulk $\mathrm{Si}$ substrates, enabling the coexistence of SiGe photonic and electronic devices on the same chip.

\section{Competing Interests}

The authors declare that they have no competing interests.

\section{Acknowledgments}

The research leading to these results was funded by the UK Engineering and Physical Sciences Research Council (EPSRC) under the grant "HERMES" and is also supported by the National Research Foundation of Singapore (NRFCRP12-2013-04). The fabrication was carried out at the Southampton Nano Centre, University of Southampton, UK. The data in this paper can be accessed at 10.5258/SOTON/ 393034 .

\section{References}

[1] J. P. Dismukes, L. Ekstrom, and R. J. Paff, "Lattice parameter and density in germanium-silicon alloys," Journal of Physical Chemistry, vol. 68, no. 10, pp. 3021-3027, 1964.

[2] R. Braunstein, A. R. Moore, and F. Herman, "Intrinsic optical absorption in germanium-silicon alloys," Physical Review, vol. 109, no. 3, pp. 695-710, 1958.

[3] J. Liu, M. Beals, A. Pomerene et al., "Waveguide-integrated, ultralow-energy GeSi electro-absorption modulators," Nature Photonics, vol. 2, no. 7, pp. 433-437, 2008.

[4] Y. Luo, X. Zheng, G. Li et al., "Strong electro-absorption in GeSi epitaxy on silicon-on-insulator (SOI)," Micromachines, vol. 3, no. 2, pp. 345-363, 2012.

[5] P. Chaisakul, D. Marris-Morini, J. Frigerio et al., "Integrated germanium optical interconnects on silicon substrates," Nature Photonics, vol. 8, no. 6, pp. 482-488, 2014.

[6] C. G. Littlejohns, Y. Hu, F. Y. Gardes et al., "50 Gb/s silicon photonics receiver with low insertion loss," IEEE Photonics Technology Letters, vol. 26, no. 7, pp. 714-717, 2014.

[7] C. G. Littlejohns, A. Z. Khokhar, D. J. Thomson et al., "Geon-Si plasma-enhanced chemical vapor deposition for low-cost photodetectors," IEEE Photonics Journal, vol. 7, no. 4, 2015.

[8] M. V. Fischetti and S. E. Laux, "Band structure, deformation potentials, and carrier mobility in strained $\mathrm{Si} \mathrm{Ge}$, and $\mathrm{SiGe}$ alloys," Journal of Applied Physics, vol. 80, no. 4, pp. 2234-2252, 1996.

[9] D. J. Thomson, H. Porte, B. Goll et al., "Silicon carrier depletion modulator with $10 \mathrm{Gbit} / \mathrm{s}$ driver realized in high-performance photonic BiCMOS," Laser \& Photonics Reviews, vol. 8, no. 1, pp. 180-187, 2014.

[10] L. Zimmermann, "High-performance photonic BiCMOSnext generation more-than-Moore technology for the large bandwidth era," ECS Transactions, vol. 64, pp. 13-18, 2014.

[11] E. P. A. M. Bakkers, J. A. van Dam, S. De Franceschi et al., "Epitaxial growth of InP nanowires on germanium," Nature Materials, vol. 3, no. 11, pp. 769-773, 2004.

[12] H. Liu, T. Wang, Q. Jiang et al., "Long-wavelength InAs/GaAs quantum-dot laser diode monolithically grown on Ge substrate," Nature Photonics, vol. 5, no. 7, pp. 416-419, 2011.

[13] A. D. Lee, Q. Jiang, M. Tang, Y. Zhang, A. J. Seeds, and H. Liu, "InAs/GaAs quantum-dot lasers monolithically grown on $\mathrm{Si}$, $\mathrm{Ge}$, and Ge-on-Si substrates," IEEE Journal on Selected Topics in Quantum Electronics, vol. 19, no. 4, article 1901107, 2013.

[14] A. Y. Hikavyy, E. Rosseel, S. K. Dhayalan et al., "(Invited) high Ge content SiGe thin films: growth, properties and integration," ECS Transactions, vol. 64, no. 6, pp. 831-839, 2014. 
[15] C. Rosenblad, H. Von Känel, M. Kummer, A. Dommann, and E. Müller, "A plasma process for ultrafast deposition of SiGe graded buffer layers," Applied Physics Letters, vol. 76, no. 4, pp. 427-429, 2000.

[16] N. Burle, S. Escoubas, E. Kasper, J. Werner, M. Oehme, and K. Lyutovich, "X-ray imaging and diffraction study of strain relaxation in MBE grown SiGe/Si layers," Physica Status Solidi (C), vol. 10, no. 1, pp. 52-55, 2013.

[17] H. Nelson, "Epitaxial growth from the liquid state and its application to the fabrication of tunnel and laser diodes," $R C A$ Review, vol. 24, pp. 603-615, 1963.

[18] R. H. Saul and D. D. Roccasecca, "Vapor-doped multislice LPE for efficient GaP green LED's," Journal of the Electrochemical Society, vol. 120, no. 8, pp. 1128-1131, 1973.

[19] H. H. Wieder, A. R. Clawson, and G. E. McWilliams, "In $\mathrm{Ga}_{1-x} \mathrm{AsyP}_{1-y} / \mathrm{InP}$ heterojunction photodiodes," Applied Physics Letters, vol. 31, pp. 468-470, 1977.

[20] M. B. Panish, I. Hayashi, and S. Sumski, "Double-heterostructure injection lasers with room-temperature thresholds as low AS 2300 A/SQ CM"," Applied Physics Letters, vol. 16, no. 8, pp. 326-327, 1970.

[21] Y. Liu, M. D. Deal, and J. D. Plummer, "High-quality singlecrystal Ge on insulator by liquid-phase epitaxy on Si substrates," Applied Physics Letters, vol. 84, no. 14, pp. 2563-2565, 2004.

[22] D. J. Tweet, J. J. Lee, J.-S. Maa, and S. T. Hsu, "Characterization and reduction of twist in Ge on insulator produced by localized liquid phase epitaxy," Applied Physics Letters, vol. 87, no. 14, Article ID 141908, 3 pages, 2005.

[23] M. Miyao, T. Tanaka, K. Toko, and M. Tanaka, "Giant Geon-insulator formation by $\mathrm{Si}$-Ge mixing-triggered liquid-phase epitaxy," Applied Physics Express, vol. 2, no. 4, Article ID 045503, 2009.

[24] M. Miyao, K. Toko, T. Tanaka, and T. Sadoh, "High-quality single-crystal Ge stripes on quartz substrate by rapid-meltinggrowth," Applied Physics Letters, vol. 95, no. 2, Article ID 022115, 3 pages, 2009.

[25] T. Hashimoto, C. Yoshimoto, T. Hosoi, T. Shimura, and H. Watanabe, "Fabrication of local ge-on-insulator structures by lateral liquid-phase epitaxy: effect of controlling interface energy between ge and insulators on lateral epitaxial growth," Applied Physics Express, vol. 2, no. 6, Article ID 066502, 2009.

[26] T. Sakane, K. Toko, T. Tanaka, T. Sadoh, and M. Miyao, "Strained single-crystal GOI (Ge on Insulator) arrays by rapid-melting growth from Si (1 111$)$ micro-seeds," Solid-State Electronics, vol. 60, no. 1, pp. 22-25, 2011.

[27] K. Toko, Y. Ohta, T. Sakane, T. Sadoh, I. Mizushima, and M. Miyao, "Single-crystalline (100) Ge networks on insulators by rapid-melting growth along hexagonal mesh-pattern," Applied Physics Letters, vol. 98, no. 4, Article ID 042101, 2011.

[28] T. Tanaka, K. Toko, T. Sadoh, and M. Miyao, "High quality single-crystalline Ge-rich $\mathrm{SiGe}$ on insulator structures by Si-doping controlled rapid melting growth," Applied Physics Express, vol. 3, no. 3, Article ID 031301, 2010.

[29] H. Y. S. Koh, Rapid melt growth of silicon germanium for heterogeneous integration on silicon [Ph.D. thesis], Department of Electrical Engineering, Stanford University, Stanford, Calif, USA, 2011.

[30] R. Matsumura, Y. Tojo, M. Kurosawa, T. Sadoh, I. Mizushima, and M. Miyao, "Growth-rate-dependent laterally graded SiGe profiles on insulator by cooling-rate controlled rapid-meltinggrowth," Applied Physics Letters, vol. 101, no. 24, Article ID 241904, 2012.
[31] N. Armour, Transport phenomena in liquid phase diffusion growth of silicon germanium [Ph.D. thesis], Department of Mechanical Engineering, University of Victoria, Victoria, Canada, 2012.

[32] Y. Tojo, R. Matsumura, H. Yokoyama et al., "High-quality formation of multiply stacked SiGe-on-insulator structures by temperature-modulated successive rapid-melting-growth," Applied Physics Letters, vol. 102, no. 9, Article ID 092102, 2013.

[33] X. Bai, C.-Y. Chen, P. B. Griffin, and J. D. Plummer, "Si incorporation from the seed into Ge stripes crystallized using rapid melt growth," Applied Physics Letters, vol. 104, no. 5, Article ID 052104, 2014.

[34] C. G. Littlejohns, M. Nedeljkovic, G. Z. Mashanovich, G. T. Reed, and F. Y. Gardes, "Silicon-germanium composition engineering for next generation multilayer devices and systems," in Proceedings of the 11th International Conference on Group IV Photonics (GFP '14), pp. 1-2, Paris, France, August 2014.

[35] C. G. Littlejohns, F. Y. Gardes, M. Nedeljkovic, G. Z. Mashanovich, and G. T. Reed, "Silicon diffusion engineering in rapid melt growth of silicon-germanium on insulator," ECS Transactions, vol. 64, no. 6, pp. 155-157, 2014.

[36] C. G. Littlejohns, M. Nedeljkovic, C. F. Mallinson et al., "Next generation device grade silicon-germanium on insulator," Scientific Reports, vol. 5, article 8288, 2015.

[37] C. G. Littlejohns, T. Dominguez Bucio, M. Nedeljkovic et al., "Towards a fully functional integrated photonic-electronic platform via a single SiGe growth step," Scientific Reports, vol. 6, Article ID 19425, 2016.

[38] D. J. Thomson, C. G. Littlejohns, S. Stankovic, M. Nedeljkovic, and S. A. Reynolds, Silicon Photonics, John Wiley \& Sons, 2015.

[39] F. Y. Gardes, T. Bucio Dominguez, C. G. Littlejohns, G. Reed, K. Debnath, and L. O'Faolain, "Group IV functionalization of low index waveguides," in Proceedings of the 16th Photonics North Conference, Montréal, Canada, May 2014.

[40] T. Tezuka, N. Sugiyama, T. Mizuno, M. Suzuki, and S.-I. Takagi, "A novel fabrication technique of ultrathin and relaxed SiGe buffer layers with high Ge fraction for sub-100 nm strained silicon-on-insulator MOSFETs," Japanese Journal of Applied Physics, vol. 40, no. 4, pp. 2866-2874, 2001.

[41] G. Taraschi, A. J. Pitera, and E. A. Fitzgerald, "Strained Si, $\mathrm{SiGe}$, and Ge on-insulator: review of wafer bonding fabrication techniques," Solid-State Electronics, vol. 48, no. 8, pp. 1297-1305, 2004.

[42] S. M. Sze, Semiconductor Devices, Physics and Technology, Wiley, New York, NY, USA, 2nd edition, 2001.

[43] R. W. Olesinski and G. J. Abbaschian, “The Ge-Si (GermaniumSilicon) system," Bulletin of Alloy Phase Diagrams, vol. 5, no. 2, pp. 180-183, 1984.

[44] University-of-Cambridge, Dissemination of IT for the Promotion of Materials Science, 2014, http://www.doitpoms.ac.uk/.

[45] D. L. Harame and B. S. Meyerson, "The early history of IBM's SiGe mixed signal technology," IEEE Transactions on Electron Devices, vol. 48, no. 11, pp. 2555-2567, 2001.

[46] P. V. Pavlov and E. V. Dobrokhotov, "Self-diffusion in liquid germanium," Soviet Physics Solid State, vol. 12, pp. 225-226, 1970.

[47] P. M. Mooney, F. H. Dacol, J. C. Tsang, and J. O. Chu, "Raman scattering analysis of relaxed $\mathrm{Ge}_{x} \mathrm{Si}_{1-x}$ alloy layers," Applied Physics Letters, vol. 62, no. 17, pp. 2069-2071, 1993.

[48] O. Pagès, J. Souhabi, V. J. B. Torres, A. V. Postnikov, and K. C. Rustagi, "Re-examination of the SiGe Raman spectra: 
percolation/one-dimensional-cluster scheme and $a b$ initio calculations," Physical Review B, vol. 86, no. 4, Article ID 045201, 2012.

[49] T. S. Perova, J. Wasyluk, K. Lyutovich et al., "Composition and strain in thin $\mathrm{Si}_{1-x} \mathrm{Ge}_{x}$ virtual substrates measured by microRaman spectroscopy and x-ray diffraction," Journal of Applied Physics, vol. 109, no. 3, Article ID 033502, 2011.

[50] J. Humlíček, M. Garriga, M. I. Alonso, and M. Cardona, "Optical spectra of $\mathrm{Si}_{x} \mathrm{Ge}_{1-x}$ alloys," Journal of Applied Physics, vol. 65, no. 7, pp. 2827-2832, 1989.

[51] H. K. Shin, D. J. Lockwood, and J.-M. Baribeau, "Strain in coherent-wave SiGe/Si superlattices," Solid State Communications, vol. 114, no. 10, pp. 505-510, 2000.

[52] J. C. Tsang, P. M. Mooney, F. Dacol, and J. O. Chu, "Measurements of alloy composition and strain in thin $\mathrm{Ge}_{x} \mathrm{Si}_{1-x}$ layers," Journal of Applied Physics, vol. 75, no. 12, pp. 8098-8108, 1994.

[53] J. Groenen, R. Carles, S. Christiansen et al., "Phonons as probes in self-organized SiGe islands," Applied Physics Letters, vol. 71, no. 26, pp. 3856-3858, 1997.

[54] C.-Y. Peng, C.-F. Huang, Y.-C. Fu et al., "Comprehensive study of the Raman shifts of strained silicon and germanium," Journal of Applied Physics, vol. 105, no. 8, Article ID 083537, 2009.

[55] E. Scheil, "Bemerkungen zur schichtkristallbildung," Zeitschrift Metallkunde, vol. 34, pp. 70-72, 1942.

[56] C. G. Littlejohns, Silicon-germanium for photonic applications [Ph.D. thesis], Optoelectronics Research Centre, University of Southampton, 2015.

[57] H. Jones, Rapid Solidification of Metals and Alloys, Institution of Metallurgists, London, UK, 1983. 

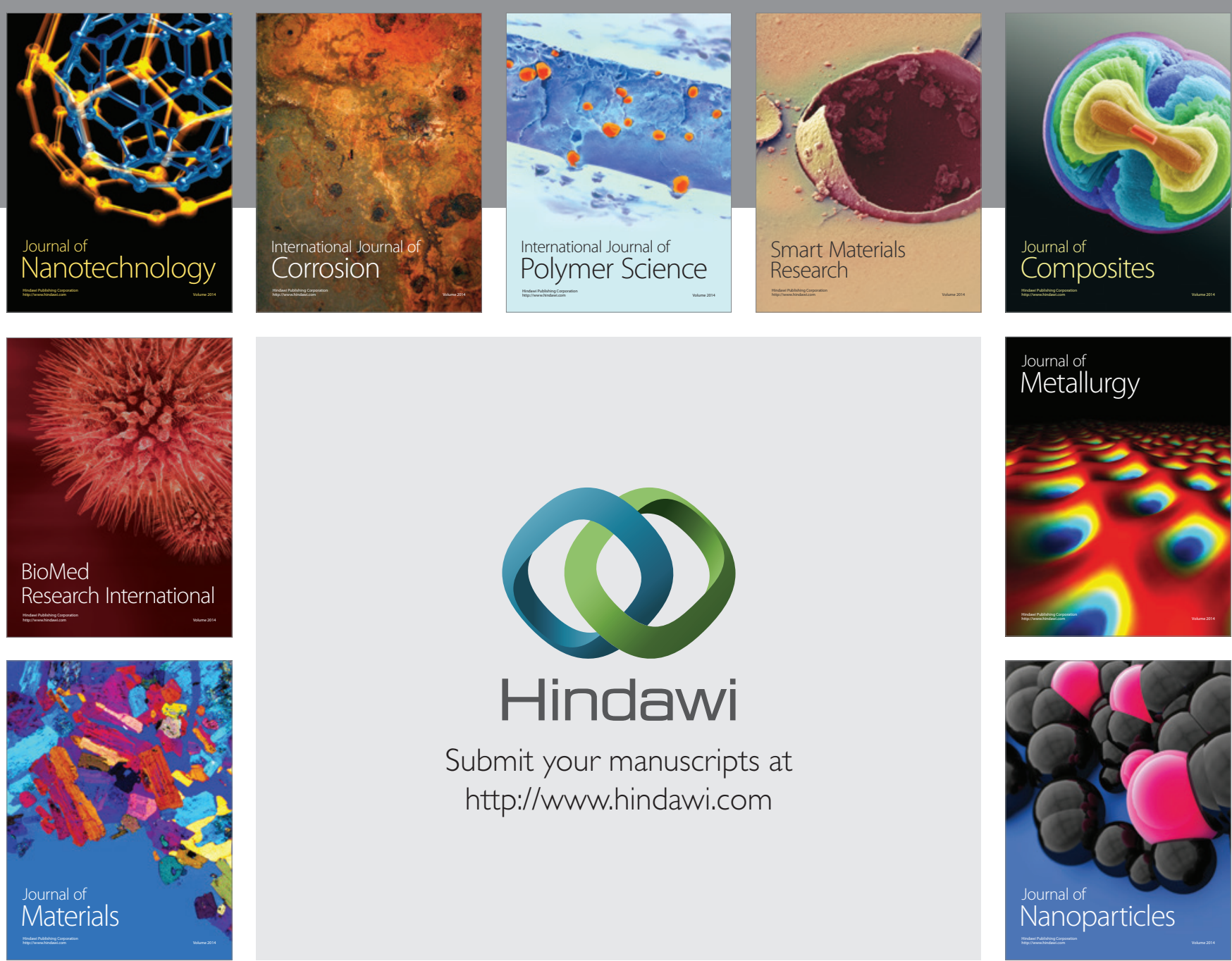

\section{Hindawi}

Submit your manuscripts at

http://www.hindawi.com

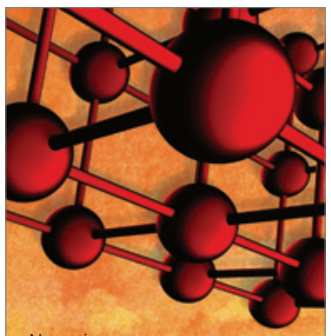

Materials Science and Engineering
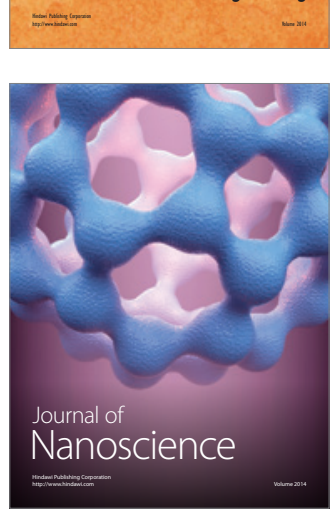
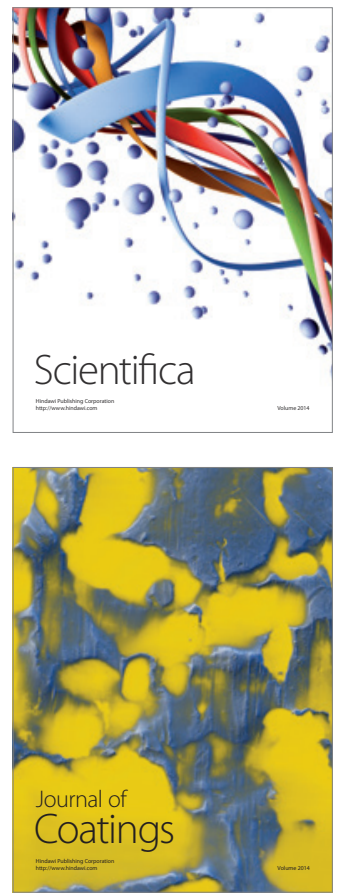
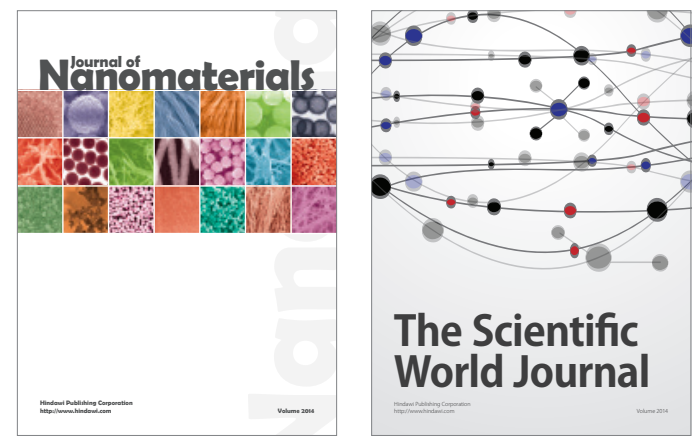

The Scientific World Journal
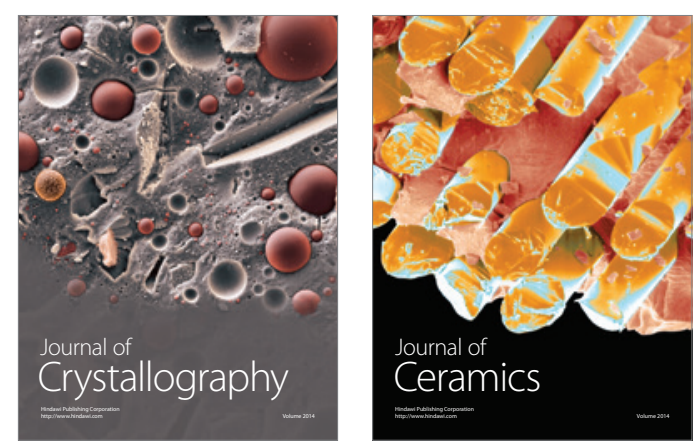
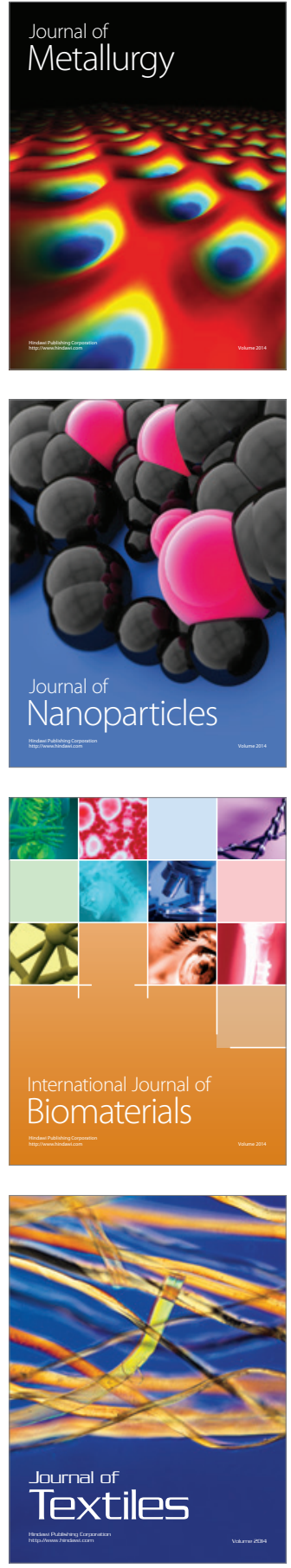